\title{
Independent signaling by Drosophila insulin receptor for axon guidance and growth
}

\author{
Caroline R. Li, Dongyu Guo ${ }^{\dagger}$ and Leslie Pick* \\ Department of Entomology and Program in Molecular and Cell Biology, University of Maryland, College Park, MD, USA
}

Edited by:

Xanthe Vafopoulou, York University,

Canada

\section{Reviewed by:}

Nick Sokol, Indiana University, USA Makio N. Takeda, Kobe University, Japan

*Correspondence:

Leslie Pick, Department of Entomology, University of Maryland, 4112 Plant Sciences Building, College Park, MD 20742, USA e-mail: Ipick@umd.edu

${ }^{\dagger}$ Present address:

Dongyu Guo, DDSR/CDER/FDA,

Silver Spring, USA
The Drosophila insulin receptor $(\mathrm{D} / \mathrm{nR})$ regulates a diverse array of biological processes including growth, axon guidance, and sugar homeostasis. Growth regulation by DInR is mediated by Chico, the Drosophila homolog of vertebrate insulin receptor substrate proteins IRS1-4. In contrast, DInR regulation of photoreceptor axon guidance in the developing visual system is mediated by the $\mathrm{SH}$ - $-\mathrm{SH} 3$ domain adaptor protein Dreadlocks (Dock). In vitro studies by others identified five NPXY motifs, one in the juxtamembrane region and four in the signaling $\mathrm{C}$-terminal tail (C-tail), important for interaction with Chico. Here we used yeast two-hybrid assays to identify regions in the DInR C-tail that interact with Dock. These Dock binding sites were in separate portions of the C-tail from the previously identified Chico binding sites. To test whether these sites are required for growth or axon guidance in whole animals, a panel of DInR proteins, in which the putative Chico and Dock interaction sites had been mutated individually or in combination, were tested for their ability to rescue viability, growth and axon guidance defects of dinr mutant flies. Sites required for viability were identified. Unexpectedly, mutation of both putative Dock binding sites, either individually or in combination, did not lead to defects in photoreceptor axon guidance. Thus, either sites also required for viability are necessary for DInR function in axon guidance and/or there is redundancy built into the DInR/Dock interaction such that Dock is able to interact with multiple regions of DInR. We also found that simultaneous mutation of all five NPXY motifs implicated in Chico interaction drastically decreased growth in both male and female adult flies. These animals resembled chico mutants, supporting the notion that DInR interacts directly with Chico in vivo to control body size. Mutation of these five NPXY motifs did not affect photoreceptor axon guidance, segregating the roles of DInR in the processes of growth and axon guidance.

Keywords: Drosophila, insulin receptor, Chico, Dock, RTK

\section{INTRODUCTION}

Receptor tyrosine kinases (RTKs) play diverse roles in development, differentiation, homeostasis and disease [reviewed in Lemmon and Schlessinger (2010)]. This diversity in biological function is exemplified by Insulin/Insulin-like signaling (IIS) pathways which have been implicated in a broad range of biological processes and diseases including diabetes, obesity, and cancer [reviewed in Nakae et al. (2001); Baserga et al. (2003); Kahn et al. (2005); Taguchi and White (2008)]. IIS impacts virtually all basic cellular processes, including transcription, translation, and cell growth, with specific effects on mitogenesis, glycogen synthesis, lipolysis, cell survival, and glucose uptake. How the different biological functions of single RTKs are executed-in different cell types, at different life cycle stages, or in response to different environmental cues-is not well understood.

Key components required to segregate the biological activities of RTKs are adapter proteins that link RTKs to discrete downstream pathways [reviewed in Kholodenko (2006); Murphy and Blenis (2006); Pawson (2007)]. Signaling is activated by ligand binding which induces a conformational change in the insulin receptor (IR), activating its kinase activity, resulting in autophosphorylation, substrate phosphorylation and the binding of adapter proteins to phosphorylated tyrosines [reviewed in Pessin and Frattali (1993); White (1998); Fisher and White (2004); Hou and Pessin (2007); Kohanski (2007); Taguchi and White (2008); Hubbard (2013)]. The insulin receptor substrates (IRS1-4) are key adapter proteins that mediate many IR downstream functions [reviewed in Taguchi and White (2008); Copps and White (2012)]. These substrates amplify receptor signals by interacting with a range of additional adapter proteins and enzymes. In addition to the IRS proteins, other receptor substrates have been identified including CBL, involved in glucose uptake (Hou and Pessin, 2007), SHC, involved in mitogenesis, and CEACAM1, involved in insulin internalization and degradation (Poy et al., 2002). Further complicating an understanding of the link between receptor activation and specific biological outcomes is the observation that different RTKs utilize many of these same adapter proteins to cause different biological "readouts" [reviewed in Lemmon and Schlessinger (2010)].

Here, we have made use of Drosophila melanogaster as a relatively simple but powerful genetic model system to investigate how a single RTK functions pleiotropically to cause distinct biological outcomes. In contrast to mammals, Drosophila harbor only one IIS-family receptor, the Drosophila insulin receptor 
(DInR) (Petruzzelli et al., 1986a,b) [reviewed in Garofalo (2002)]. DInR is similar in protein sequence to both human IR and IGF$1 \mathrm{R}$ ( $\sim 26 \%$ and $\sim 27 \%$ identity respectively and $\sim 39 \%$ similarity) with highest similarity in the intracellular kinase domain, but was shown to autophosphorylate only in response to insulin (Fernandez-Almonacid and Rosen, 1987). Loss-of-function dinr mutations cause recessive lethality, demonstrating that DInR is essential for viability. Animals transheterozygous for combinations of dinr hypomorphic alleles are viable, but growth delayed and small (Fernandez et al., 1995; Chen et al., 1996; Bateman and McNeill, 2004; Colombani et al., 2005; Shingleton et al., 2005). DInR controls growth in a cell autonomous fashion: overexpression of DInR in the eye resulted in eye overgrowth due to increases in both cell size and cell number (Coelho and Leevers, 2000; Brogiolo et al., 2001; Leevers, 2001; Goberdhan and Wilson, 2003). These effects on growth are also seen for Chico, the Drosophila IRS1-4 homolog that acts downstream of DInR (Bohni et al., 1999). DInR and other IIS pathway members have been implicated in other biological processes including, but not limited to, regulation of lifespan and aging (Tatar et al., 2001; Hwangbo et al., 2004; Tatar, 2004; Mair et al., 2005; Piper et al., 2005, 2007; Piper and Partridge, 2007; Giannakou et al., 2008; Min et al., 2008; Partridge, 2008), locomotor activity (Belgacem and Martin, 2006), eating behavior (Wu et al., 2005; Lingo et al., 2007), oogenesis (Tatar et al., 2001; LaFever and DrummondBarbosa, 2005; Hsu et al., 2008), heart function (Ocorr et al., 2007), nutrient sensing (Britton et al., 2002; Puig and Tjian, 2006), and metabolism, with surviving dinr transheterozygotes or heterozygotes displaying increased levels of whole body or circulating sugar (Shingleton et al., 2005; Belgacem and Martin, 2006).
DInR is highly expressed in the developing nervous system and we previously found that it is required for photoreceptor axon guidance (Song et al., 2003). In this previous study, we used a yeast two-hybrid screen to identify DInR intracellular partners. As bait for this screen, we used the intracellular portion of DInR, which autophosphorylated in yeast cells. This screen identified Dreadlocks (Dock) as a DInR partner. Dock had previously been shown to be required for photoreceptor axon guidance during development of the adult visual system in Drosophila (Garrity et al., 1996), suggesting that DInR might also play a role in this process. Our yeast two-hybrid assays showed that interaction with Dock requires DInR kinase activity and involves both the SH2 and the SH3 domains of Dock. This finding was consistent with in vivo rescue experiments showing that both $\mathrm{SH} 2$ and $\mathrm{SH} 3$ domains of Dock are required for photoreceptor axon guidance (Rao and Zipursky, 1998). Using the eyFLP-FRT system (Newsome et al., 2000) to generate homozygous dinr mutant tissue in a heterozygous background, we found that photoreceptor axon guidance was disrupted in these dinr mosaic animals. Whole animal dinr transheterozygotes showed similar, but more extreme defects, similar to defects seen in dock mutants. In contrast, animals carrying chico mitotic clones or whole animal chico mutants showed normal patterns of photoreceptor axon targeting. Since chico cells are small, similar to dinr clones, this result shows that the axon guidance defects seen for dinr mosaics are not a simple result of dinr-associated growth defects.

On the basis of these results, we previously proposed (Song et al., 2003) that the roles of DInR in growth and axon guidance are independent and mediated by different adapter proteins: binding to Dock regulates axon guidance while binding to Chico controls growth (Figure 1A). DInR interaction with either Dock

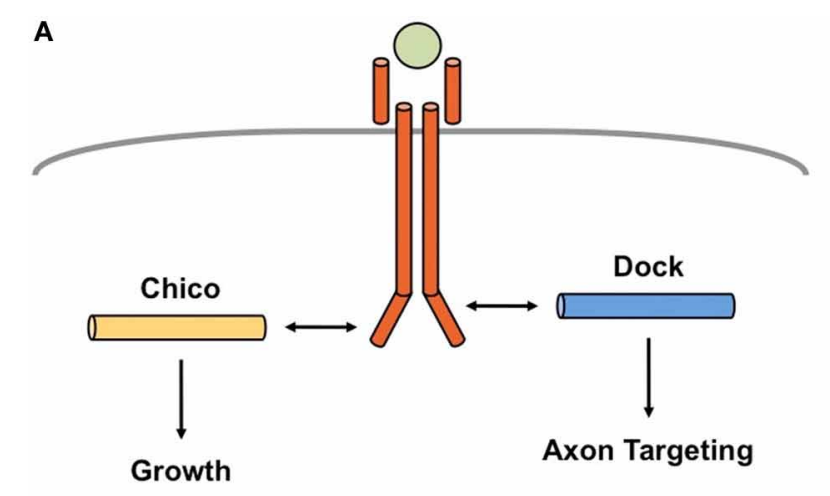

B

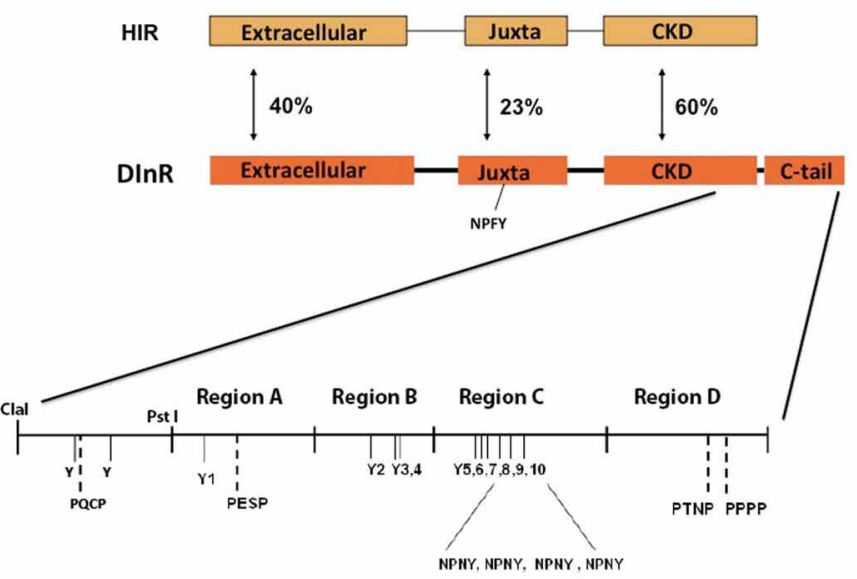

FIGURE 1 | DInR signals independently through Chico and Dock to control growth and axon guidance. (A) We and others proposed that DInR, after DILP binding, signals independently through Chico to control growth and Dock to control axon targeting. Panel modified from Dickson (2003). (B) Schematic of DInR sequence with candidate binding regions indicated. The C-tail of DInR, previously shown to be required for interaction with Dock

(Song et al., 2003), was divided into four regions (Regions A-D) for analysis. Dock is expected to interact with tyrosine residues $(\mathrm{Y})$ via its $\mathrm{SH} 2$ domain and PXXP residues via its SH3 domains. Four NPNY sites in the C-tail and the juxtamembrane NPFY (NPXY-1351) were required for interaction with Chico in cell-based assays (Poltilove et al., 2000). All tyrosine residues in the C-tail are indicated in the figure. 
(Song et al., 2003) or Chico (Poltilove et al., 2000) in vitro requires the DInR C-terminal tail (C-tail), an extension absent in mammalian IR/IGF-1R (Fernandez et al., 1995; Ruan et al., 1995; Yenush et al., 1996). This C-tail contains multiple potential tyrosine phosphorylation sites and is required for DInR signaling in cell culture (Fernandez et al., 1995; Ruan et al., 1995; Yenush et al., 1996; Marin-Hincapie and Garofalo, 1999). The C-tail also contains YXXM motifs that mediate direct binding to PI3K in cell culture (Yenush et al., 1996), but rescue experiments in flies suggested that Chico is necessary to link DInR to PI3K for signaling and growth control in vivo (Oldham et al., 2002). Five NPXY motifs, one in the juxtamembrane region and four in the DInR C-tail, were shown to be important for interaction with Chico in vitro (Poltilove et al., 2000). Here, we used yeast two-hybrid assays to identify the regions of DInR that bind Dock. We found that the region of the DInR C-tail that binds Dock is distinct and separable from the region containing Chico interaction sites. We show that rescue of viability of dinr mutants requires kinase activity and one specific tyrosine residue in the C-tail. In contrast, a DInR protein carrying mutations in all Chico interaction sites rescued viability and axon guidance defects, but yielded growth defects similar to those seen in chico mutants. Finally, DInR proteins carrying mutations in identified Dock binding sites still rescued axon guidance defects, suggesting a high degree of redundancy for this function of DInR.

\section{MATERIALS AND METHODS CONSTRUCTION OF dinr cDNA}

A full-length dinr cDNA was assembled from genomic fragments as follows: a $7.4 \mathrm{~kb}$ genomic EcoRI-NheI fragment spanning the entire dinr coding region and including $770 \mathrm{bp}$ of $5^{\prime}$ UTR, 9 introns and $191 \mathrm{bp}$ of $3^{\prime}$ UTR was isolated from BAC48I01 (BACPAC, Oakland, CA). Subsequently, this EcoRI-NheI fragment was inserted into $p S P-l u c^{+} N F$ (Promega, Madison, WI) to generate plasmid pSPgdinr. An EcoRI-KpnI fragment including exons 1-4 from pSPgdinr was inserted into pSP72 (Promega, Madison, WI) generating plasmid pSPgdinrEK and introns 1, 2 , and 3 were deleted sequentially by PCR $\left(95^{\circ} \mathrm{C}\right.$ for $1 \mathrm{~min}$; 30 cycles of: $95^{\circ} \mathrm{C}$ for $30 \mathrm{~s}, 55^{\circ} \mathrm{C}$ for $30 \mathrm{~s}$ and $72^{\circ} \mathrm{C}$ for $6 \mathrm{~min}$; $72^{\circ} \mathrm{C}$ for $\left.10 \mathrm{~min}\right)$. PCR products were self-ligated after gel purification with GenElute agarose spin columns (Sigma, St. Louis, $\mathrm{MO})$ to generate plasmid $p S P \operatorname{din} r E K$. Similarly, to remove introns 4-9, a KpnI-AflII fragment from $p S P g d i n r$ was inserted into pSP72 to generate pSPgdinrKAf. Introns 4-7 were eliminated by replacing the KpnI-NsiI fragment of pSPgdinrKAf with a corresponding KpnI-NsiI cDNA fragment of dinr generated by RT-PCR using a SuperScript One-Step RT-PCR kit (Invitrogen, Carlsbad, CA). Total RNA for this reaction was isolated from 0 to $10 \mathrm{~h} w^{1118}$ fly embryos using Trizol reagent (GibcoBRL, Carlsbad, CA). Introns 8 and 9 were deleted from pSPgdin$r K A f$ by PCR, as described above. Together, these steps generated plasmid pSPdinrMid, which contains the KpnI-AflII coding region, lacking all introns. To generate a full-length dinr cDNA, the KpnI site outside the dinr gene in pSPgdinr was deleted by digestion with NheI and AatII, followed by a fill-in reaction with Klenow. Genomic EcoRI-KpnI and KpnI-AflII fragments were replaced by cDNA fragments from $p S P$ dinrEK and pSPdinrMid, respectively, to generate $p S P d i n r$, the final construct.
Sequencing of pSPdinr confirmed that it matched the BDGP sequence FBgn0013984. We note that plasmids containing partial or full-length dinr cDNA were extremely unstable and could only be maintained in One Shot ${ }^{\circledR}$ TOP10 cells (Invitrogen, Carlsbad, CA).

\section{CONSTRUCTION OF MUTANT dinr cDNAs}

To test the function of regions of DInR in intracellular signaling and rescue of dinr mutant phenotypes, cDNAs were generated that were truncated or that carried specific point mutations in candidate adapter binding sites. To generate deletions, the AflIIStuI fragment from $p S P d i n r$ was replaced by PCR amplification of pSPdinr of the fragment of interest. Borders of the C-tail and deletions are shown schematically in Figure 1B. The start of the C-tail is at position Q1672. Deletions were generated to target large regions including tyrosine and PXXP residues that are candidate protein interaction motifs in the C-tail. Deletions were generated by standard PCR reactions and removed the following regions: $\triangle \mathrm{AB}$ removed amino acids D1611 to K1825; $\triangle \mathrm{CD}$ removed $\mathrm{S} 1860$ to $\mathrm{P} 2131 ; \Delta \mathrm{D}$ removed $\mathrm{D} 2060$ to $\mathrm{P} 2131$; $\triangle \mathrm{A}$ removed $\mathrm{D} 1611$ to $\mathrm{L} 1742 ; \triangle \mathrm{BCD}$ removed $\mathrm{T} 1760$ to $\mathrm{P} 2131$; $\triangle \mathrm{ABC}$ removed $\mathrm{D} 1611$ to $\mathrm{S} 2042$. Note that $\triangle \mathrm{A}$ constructs removed a portion of the kinase domain, beginning at D1611, in addition to the A region. Also, for constructs missing the D region, the C-terminal end of DInR, from amino acids R2132 to $\mathrm{A} 2144$, is present upstream of the MYC3 tag. This region includes a PPPP sequence (P2133-P2136). The following point mutations were generated: DInR-KA $(\mathrm{K} 1405 \rightarrow \mathrm{A})$ in the kinase domain; $\mathrm{Y} 1 \mathrm{~F}(\mathrm{Y} 1714 \rightarrow \mathrm{F})$ in the $\mathrm{C}$-tail; $\mathrm{Y} 2 \mathrm{~F}(\mathrm{Y} 1776 \rightarrow \mathrm{F})$ in the $\mathrm{C}$ tail; $\mathrm{Y} 3,4 \mathrm{~F}(\mathrm{Y} 1790 \rightarrow \mathrm{F}, \mathrm{Y} 1793 \rightarrow \mathrm{F})$ in the $\mathrm{C}$-tail; LESL $(\mathrm{P} 1724 \rightarrow \mathrm{L}$, $\mathrm{P} 1727 \rightarrow \mathrm{L})$ in the C-tail; $\mathrm{Y7F}(\mathrm{Y} 1965 \rightarrow \mathrm{F}), \mathrm{Y} 8 \mathrm{~F}(\mathrm{Y} 1989 \rightarrow \mathrm{F})$, $\mathrm{Y} 9 \mathrm{~F}(\mathrm{Y} 2005 \rightarrow \mathrm{F}), \mathrm{Y} 10 \mathrm{~F}(\mathrm{Y} 2026 \rightarrow \mathrm{F})$ and combinations thereof, in NPXY sites in the C-tail. To generate these point mutations, a PCR product of the dinr C-tail from plasmid DInRCKD-PAS21 (Song et al., 2003) was subcloned into the vector $p S P 72$ at its ClaI/KpnI site to yield $p S P$ dinrMT. Site-directed mutagenesis of pSPdinrMT was done by PCR $\left(95^{\circ} \mathrm{C}\right.$ for $2 \mathrm{~min}$; 18 cycles of: $95^{\circ} \mathrm{C}$ for $30 \mathrm{~s}, 55^{\circ} \mathrm{C}$ for $1 \mathrm{~min}$ and $68^{\circ} \mathrm{C}$ for $8 \mathrm{~min}$ ). The PCR product was digested with DpnI for $1 \mathrm{~h}$ at $37^{\circ} \mathrm{C}$ to destroy any unmutagenized template plasmid present, and was transformed into XL-1 competent cells. All mutations were confirmed by DNA sequencing. The ClaI-KpnI fragments with various dinr mutations were shuttled from $p S P$ dinrMT into $p A S 2-1 O F C T$ for yeast two-hybrid assays. Primer sequences available upon request.

To generate transgenic Drosophila, full-length, partial or point mutation-containing dinr cDNAs were inserted into pUASTdinrMYC3, a P-element vector which includes a 102 bp region encoding a $3 \mathrm{X}$ Myc tag to generate in-frame C-terminal fusions. This vector was generated as follows. The AflII-NheI fragment in pSPdinr was replaced by the PCR fragment of $p S P d i n r$ amplified using two primers, P51 and Srf2, and digested with AflII/NheI to introduce an SrfI site so that the MYC3 tag could be inserted into the vector. The newly generated plasmid was named pSPdinrM. A MYC3 tag was excised from the plasmid $p S R L$ hSNT MYC3 (a gift from Dr. Mitch Goldfarb, Hunter College) and subcloned into the SrfI/NotI site of $p S P \operatorname{din} r M$, generating pSPdinrMYC3. The dinrMYC3 cDNA from $p S P$ dinrMYC3 was subcloned into the EcoRI/NotI site of pUAST to generate the 
full-length $p U A S T$ dinrMYC3 plasmid. dinr cDNAs carrying deletions $(\triangle \mathrm{ABC}, \triangle \mathrm{AB}, \triangle \mathrm{CD}$ ) were inserted into pUASTdinrMYC3 by replacing the BsiWI-NotI fragment of $p U A S T$ dinrMYC3. Point mutations in the C-tail of dinr, generated in pSPdinrMT, were moved into pUASTdinrMYC3 by the replacement of the AflIINotI fragment.

To test the one NPFY motif in the juxtamembrane region, pUASTdinr(JM-NPFF)MYC3 was generated, in which the tyrosine in the juxtamembrane NPFY motif was changed to a phenylalanine $(\mathrm{Y} 1354 \rightarrow \mathrm{F})$. Site-directed mutagenesis to change the TAT codon for tyrosine to the TTT codon for phenylalanine was carried out with standard methods using pSPdinrMYC3 and Vent polymerase (NEB, Ipswich, MA). Then, a $\sim 7 \mathrm{~kb}$ fragment spanning the entire dinrMYC3 coding region, and thus containing the mutated juxtamembrane NPFF site, was released from the mutated pSPdinrMYC3 plasmid with NotI and EcoRI; this fragment was inserted into the NotI and EcoRI sites of pUAST-dinr(Y7,8,9,10F)MYC3, replacing the entire $\operatorname{dinr}(Y 7,8,9,10 F) M Y C 3$ coding region. pUASTdinr(5NPXF)MYC3 was then made by excising a $\sim 4 \mathrm{~kb}$ fragment containing the 4 mutated NPXF sites in the C-tail from pUASTdinr $(Y 7,8,9,10 F) M Y C 3$ using AflII and inserting it into the AflII site of $p U A S T \operatorname{din} r(J M-N P F F) M Y C 3$ to replace the AflII fragment. The orientation and sequence of each dinr variant was verified by sequencing.

\section{YEAST TWO-HYBRID ASSAYS}

Yeast two-hybrid assays were performed as described (Song et al., 2003) using $p A S 2-1 O F C T$ carrying either wild type or mutant versions of DInR and pACT-Dock (Song et al., 2003). One hundred microliters of saturated culture was inoculated into $3 \mathrm{ml}$ of fresh media and grown to mid-log phase. One milliliter of culture was spun down. The pellet was suspended completely in $200 \mu \mathrm{l}$ of $10 \mathrm{mM}$ Tris $(\mathrm{pH} 7.5) / 0.05 \%$ Triton X-100. The samples were stored at $-80^{\circ} \mathrm{C}$. Frozen samples were allowed to thaw slowly on ice before analysis. One milliliter of ONPG solution was added and mixed by inverting several times. The reaction was carried out at $30^{\circ} \mathrm{C}$. When the color changed to medium-dark yellow, the reaction was stopped by adding $500 \mu \mathrm{l} 1 \mathrm{M} \mathrm{Na}_{2} \mathrm{CO}_{3}$ and $\mathrm{OD}_{420}$ was measured. The $\beta$-galactosidase activity was calculated with the formula: $\beta$-Gal units $=\left[\mathrm{OD}_{420}\right.$ (absorbance by reaction product $) \times 1000] /\left[\mathrm{OD}_{600}(\right.$ sample cell density $) \times(1 \mathrm{ml}) \times t$ (time of reaction)]. Assays were repeated at least three independent times using at least 3 samples for each point in each assay.

\section{GENETICS AND PHENOTYPIC ANALYSIS}

Transgenic flies were generated by Rainbow Transgenic Flies, Inc. (Camarillo, CA) by standard P-element mediated transformation. Multiple independent transgenic lines were generated for each construct whenever possible. Transgenic lines carrying insertions on chromosome II were used for rescue experiments. Transgenic lines for rescue experiments expressed DInR proteins at similar levels, determined using a modification of the method of Ronshaugen et al. (2002).

Genetic crossing schemes used to generate stocks for the dinr rescue experiments are available upon request. For the following experimental crosses, parental flies were removed as necessary to prevent overcrowding of the progeny to be used for analysis. For the lethality rescue analysis, armGAL4/arm-GAL4;FRT82Bdinr ${ }^{273} /$ TM3Sb, armGFP virgin females were crossed to UAS-X/(UAS-X or CyO);dinr ${ }^{\text {ex } 15} /$ TM3Sb, arm GFP males. Adult progeny that had eclosed were scored for their bristle phenotype: either $S b$ or non- $S b$. In the case that the UAS-X construct to be tested was homozygous lethal and had to be used in crosses with a $\mathrm{CyO}$ balancer, only non- $\mathrm{CyO}$ eclosed adult progeny were scored for their bristle phenotype.

For the growth defect rescue analysis, arm-GAL4/armGAL4;FRT82Bdinr ${ }^{273} /$ TM3Sb, armGFP virgin females were crossed to UAS-X/(UAS-X or CyO);dinrex15/TM3Sb,armGFP males. Eclosed non-Sb, non-CyO adult male or female progeny were collected separately in fresh food vials and were individually weighed in an ATI Cahn C-33 microbalance approximately 3-18 days after eclosion.

For the photoreceptor axon guidance rescue analysis, armGAL4/arm-GAL4;FRT82Bdinr ${ }^{273} /$ TM6BTb,GFP virgin females were crossed to UAS-X/UAS-X; dinrex15/TM6BTb,GFP males. Non-Tubby progeny at the wandering third instar larval or white prepupal stages were analyzed.

For SEM studies, adult flies were decapitated. Heads were fixed in $2.5 \%$ glutaraldehyde overnight at $4^{\circ} \mathrm{C}$, washed $3 \times 30 \mathrm{~min}$. with $0.1 \mathrm{M}$ PBS, dehydrated in ascending acetone grades and then critical point dried. They were then mounted on studs in the desired orientation under a stereo binocular microscope and coated with gold (thickness $30-35 \mathrm{~nm}$ ). Scanning was done on SEM mode in an AMRAY $1820 \mathrm{D}$ electron microscope at $15 \mathrm{kV}$.

\section{PHOTORECEPTOR AXON GUIDANCE ANALYSIS}

Eye-brain complexes were dissected from third instar larvae or white prepupae in phosphate-buffered saline (PBS). A standard protocol kindly provided by $\mathrm{C}$. H. Lee was generally followed for the staining of eye-brain complexes with monoclonal antibody 24B10 (MAb24B10): eye-brain complexes were fixed in $2 \%$ paraformaldehyde in a lysine-phosphate buffer containing $0.25 \%$ sodium m-periodate, washed in $0.5 \%$ Triton-X-100 in PBS (PBT), blocked in 10\% normal goat serum (NGS) in PBT, incubated in 1:200 MAb24B10 in 10\% NGS in PBT at $4^{\circ} \mathrm{C}$ overnight or longer, washed in PBT, incubated in 1:200 HRPconjugated goat anti-mouse antibody in 10\% NGS in PBT at room temperature for at least $2 \mathrm{~h}$, washed in PBT, incubated in $\mathrm{DAB}$, washed in PBS, and cleared and mounted in $70 \%$ glycerol in PBS. MAb24B10 specifically stains the cell bodies and axonal membranes of differentiated photoreceptors in Drosophila melanogaster and was originally generated by Zipursky et al. (1984). MAb24B10 used in our experiments was purchased from the Developmental Studies Hybridoma Bank at The University of Iowa.

\section{RESULTS}

\section{THE DInR C-TAIL HARBORS SEPARATE BINDING SITES FOR DOCK AND CHICO}

As described above, we proposed that DInR signals independently through Dock and Chico to regulate axon guidance and growth, respectively (Figure 1A). To test this, yeast two-hybrid assays $(\mathrm{Y} 2 \mathrm{H})$ were used to identify potential Dock interaction sites in DInR. Because Dock interaction with DInR required the C-tail (Song et al., 2003), a series of small deletions and 
point mutations in DInR was generated in this portion of DInR (Figure 1B; Materials and Methods). For the deletion series, the C-terminal portion of DInR was divided arbitrarily into 4 regions (Regions A-D, Figure 1B) which were fused to the rest of the intracellular domain of DInR to allow for autophosphorylation in yeast (see Song et al., 2003). Region A includes a portion of the highly conserved kinase domain (between the ClaI and PstI sites indicated in Figure 1B), as well as the N-terminal portion of the C-tail that harbors two potential Dock interaction sites, Y1714 and a PESP motif at position 1724. Region B harbors three tyrosines (Y1776,1790,1793) that could potentially interact with Dock. Region $\mathrm{C}$ includes 6 tyrosines, the last four of which are embedded within NPXY consensus sequences previously shown to be involved in Chico interaction (Poltilove et al., 2000). Finally, region D contains one PXXP sequence, potentially able to bind Dock's SH3 domains. These tyrosine residues, all indicated in Figure 1B, are the only tyrosine residues present in the DInR C-tail.

As shown in Figure 2, the full-length DInR intracellular domain interacted strongly with Dock. DInR- $\Delta \mathrm{D}$, which lacks the $\mathrm{D}$ region, and DInR- $\triangle \mathrm{CD}$, which lacks both $\mathrm{C}$ and $\mathrm{D}$ regions, interacted as strongly with Dock as full-length DInR. This result suggests that regions A and B are sufficient for the DInR/Dock interaction. Consistent with this, proteins lacking the A (DInR$\Delta \mathrm{A}$ ) or $\mathrm{A}$ and $\mathrm{B}$ (DInR- $\triangle \mathrm{AB}$ ) regions did not interact detectably with Dock. However, the A region alone was not sufficient for interaction, as DInR- $\triangle \mathrm{BCD}$ did not interact detectably with Dock. Note that the deletion of the A (DInR- $\Delta \mathrm{A}$ ) region alone suggests that the $\mathrm{B}$ region is also not sufficient for Dock interaction; however, as conserved regions of the kinase domain were removed in DInR- $\Delta \mathrm{A}$, we cannot make a firm conclusion about the $\mathrm{C}$-tail requirements in this case.

To further investigate the sequence motifs necessary for DInR/Dock interaction, point mutations were generated in tyrosine residues in candidate adapter protein binding sites in the C-tail. As shown in Figure 2, mutation of Y1714 to F (DInR$\mathrm{Y} 1 \mathrm{~F})$ in region A did not significantly decrease interaction with Dock. In contrast, mutations of Y1776 in region B (DInR-Y2F) greatly decreased Dock binding. Mutations of the other tyrosine

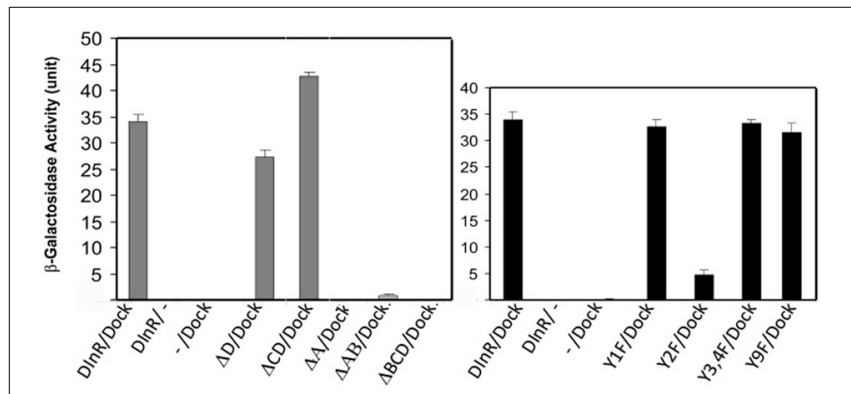

FIGURE 2 | Yeast two-hybrid assays identify candidate Dock interaction sites in the DInR C-tail. pACT-Dock was tested for interaction with pAS2.1 containing the DInR intracellular domain or variants thereof, as indicated. $\beta$-galactosidase activity is shown. Negative controls: pAS2.1-DInR with empty pACT (-); empty pAS2.1 (-) with pACT-Dock. Error bars indicate standard deviation. residues in the B region (double mutation of Y1790 and Y1793; DInR-Y3,4F) did not alter Dock interaction. Finally, point mutations of the tyrosine residues in the $\mathrm{C}$ region had modest or no effect on Dock interaction, shown here for Y2005F (DInR-Y9F). Together, these results suggested that DInR interaction with Dock requires the B region, in particular, Y1776 in this portion of DInR. Since the A region was also required for Dock interaction, but the tyrosine in this region was not, and since Dock function in vivo involves both SH2 and SH3 domains (Rao and Zipursky, 1998), these results suggested that the PXXP motif in the A region also interacts with Dock.

\section{FULL-LENGTH Myc-TAGGED DInR PROMOTES GROWTH AND RESCUES VIABILITY}

Based upon the findings summarized above, we generated a series of transgenes carrying mutations or deletions of sites critical for kinase activity or adapter protein binding in the context of the full-length dinr coding region (Figure 1B, Table 1). A C-terminal $3 x$ Myc tag was added to each dinr variant for antibody detection and each dinr cDNA was inserted into a $p U A S T$ vector for expression with the GAL4/UAS system (Brand et al., 1994). After P-element mediated transformation, multiple independent transformant lines were obtained for each transgene and expression levels were quantitated. To verify the function of full-length DInR in tissue growth, and to test whether the 3xMyc tag interferes with DInR function, DInR-MYC3 was expressed in the developing eye using a moderate $e y$-GAL4 driver. This resulted in cell autonomous overgrowth of the eye (Figures 3A-F). The increased eye volume resulted in eyes occupying larger volumes with increased depth, not always clearly depicted in twodimensional images of whole eyes. This eye size difference appeared to be due to an increase in both cell size and cell number. In contrast, and as expected, a DInR transgene carrying a point mutation in the ATP binding site ("kinase-dead" mutation; DInR-K1405A) appeared to function as a dominant negative in these experiments, resulting in smaller eyes (Figures 3G-I).

Table 1 | Rescue of adult lethality by DInR transgenes.

\begin{tabular}{llll}
\hline Transgene expressed & $\begin{array}{l}\text { Rescued } \\
\text { dinrex15/273 } \\
\text { adults } \\
\text { (\% non-Sb) }\end{array}$ & $\begin{array}{l}\text { dinr } \\
\text { dinr } r^{273} /+ \text { adults } \\
\text { (\% Sb) }\end{array}$ & $\begin{array}{l}\text { Total no. of } \\
\text { flies scored }\end{array}$ \\
& &
\end{tabular}

$\begin{array}{lrrr}\text { UAS-lacZ } & 0 & 100 & 237 \\ \text { UAS-dinr } & 37 & 63 & 785 \\ \text { UAS-dinr-KA } & 0 & 100 & 34 \\ \text { UAS-dinr- } C D & 27 & 73 & 271 \\ \text { UAS-dinr- } \triangle A B & 0 & 100 & 242 \\ \text { UAS-dinr-Y1F } & 0 & 100 & 245 \\ \text { UAS-dinr-Y2F } & 8 & 92 & 153 \\ \text { UAS-dinr-LESL } & 42 & 58 & 177 \\ \text { UAS-dinr-LESL,Y2F } & 11 & 89 & 254 \\ \text { UAS-dinr-JM-NPFF } & 24 & 76 & 213 \\ \text { UAS-dinr-Y9F } & 16 & 84 & 145 \\ \text { UAS-dinr-Y7,8,9,10F } & 11 & 89 & 235 \\ \text { UAS-dinr-5NPXF } & 21 & 79 & 246\end{array}$




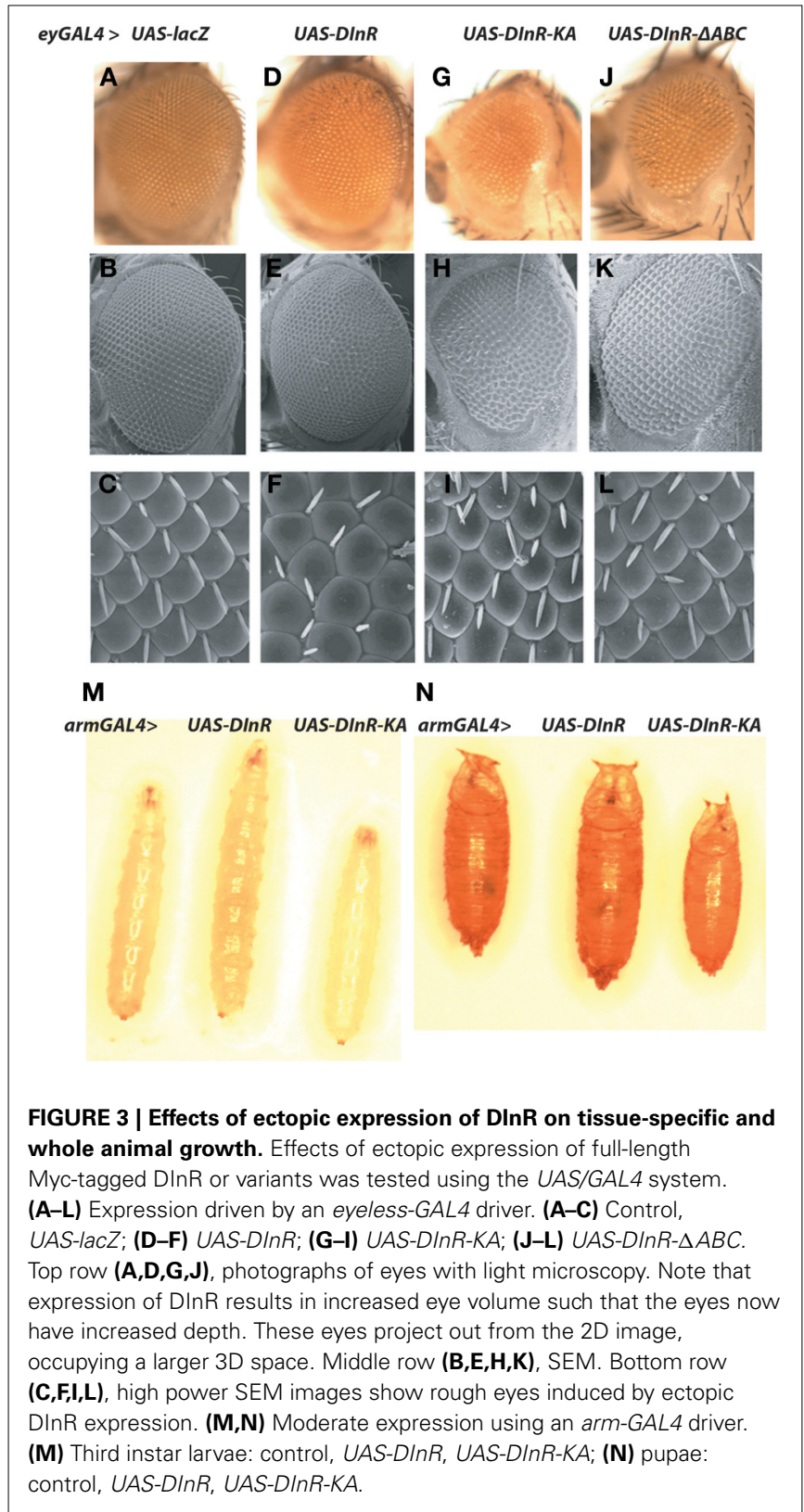

Similarly, expression of DInR- $\triangle \mathrm{ABC}$ resulted in smaller eyes (Figures 3J-L).

Overexpression of full-length DInR in a wild type background with a moderate ubiquitous driver, arm-GAL4, caused whole animal overgrowth evident at larval and pupal stages (Figures 3M,N). Expression of DInR-KA acted as a dominant negative on whole animal growth.

To test the ability of DInR transgenes to complement wild type functions of DInR, transgenes were expressed in a dinr $r^{e x 15 / 273}$ mutant background under the control of an armGAL4 driver. These dinrex15/273 transheterozygotes carry one copy of the dinr ${ }^{e x 15}$ null allele and one copy of the dinr ${ }^{273}$ weak hypomorphic allele. To test for rescue, arm-GAL4/arm-GAL4; FRT82Bdinr ${ }^{273} / T M 3 S b$, arm GFP virgin females were crossed to
UAS-X/(UAS-X or CyO);dinr ex15/TM3Sb,armGFP males and rescue of lethality was scored by counting non-Sb adults. As shown in Table 1, a negative control protein, $\beta$-galactosidase, encoded by the UAS-lacZ transgene, failed to rescue viability, as expected. However, viability to adulthood was fully rescued by wild type DInRMYC3 (UAS-dinr). UAS-dinr-KA, encoding a "kinase-dead" receptor, failed to rescue viability, consistent with the expectation that kinase activity is necessary for DInR function in vivo.

\section{RESCUE OF dinr-ASSOCIATED LETHALITY BY DInR MUTANT TRANSGENES}

To test the importance of the Dock and Chico binding sites identified in vitro (Figure 2 and (Poltilove et al., 2000)) for DInR function in vivo, the rescue approach described above was used. Mutations were introduced into full-length pUASTDInRMYC3, as described in the Materials and Methods. The mutant proteins were designed to test in vivo requirements for different DInR sequences: (1) DInR-KA, mutation of K1405 to A in the ATP binding site ("kinase-dead"). (2) DInR- $\triangle C D$, deletion of the $\mathrm{C}$ and most of the $\mathrm{D}$ regions of the C-tail. (3) DInR- $\triangle A B$, deletion of the $\mathrm{A}$ and $\mathrm{B}$ regions of the $\mathrm{C}$-tail and part of the adjoining kinase domain, $\mathrm{N}$-terminal of the C-tail. (4) DInR-Y1F, mutation of Y1714 in the A region of the C-tail. (5) DInR-Y2F, mutation of Y1776 in the B region of the C-tail. (6) DInR-LESL was designed to test the role of the potential SH3 binding PXXP motif in region A of the DInR C-tail. (7) DInR-LESL, Y2F, mutation of the PXXP in the A region and of Y1776 in the B region. (8) DInR-JM-NPFF, mutation to F of Y1354, embedded in an NPFY motif in the juxtamembrane region, previously shown to be required for Chico interaction (Poltilove et al., 2000). (9) DInR-Y9F, mutation of one of four Chico binding sites in the $\mathrm{C}$ region of the C-tail. (10) DInR-Y7,8,9,10F, simultaneous mutation of all four Chico binding sites in the $\mathrm{C}$ region of the $\mathrm{C}$-tail. (11) DInR-5NPXF (JM-NPFF,Y7,8,9,10F), simultaneous mutation of the juxtamembrane NPFY and the four Chico binding sites in region $\mathrm{C}$ of the $\mathrm{C}$-tail. dinr cDNAs encoding the DInR proteins described above were inserted into the P-element vector $p U A S T$. Multiple independent transformant lines were generated for each and insertions on chromosome II were selected for rescue experiments. DInR proteins were expressed with the GAL4/UAS system, using a moderate, ubiquitous arm-GAL4 driver.

As shown in Table 1, the CD region of the C-tail was not required for rescue to adulthood, as UAS-dinr- $\triangle C D$ expression rescued viability. In contrast, the $\mathrm{AB}$ region, containing the $\mathrm{N}$-terminal half of the $\mathrm{C}$-tail and a small portion of the conserved kinase domain, was required to support viability, as no adults were observed when UAS-dinr- $\triangle A B$ was expressed in the dinr ${ }^{e x 15 / 273}$ mutant background. Interestingly, DInR-Y1F completely failed to rescue adult lethality of dinr ${ }^{\text {ex15/273 }}$ transheterozygotes, indicating that tyrosine 1714 , within the A region of the C-tail, is crucial for adult viability. Expression of UASdinr-Y2F rescued a small number of animals, suggesting that this residue contributes to but is not absolutely required for viability. The PESP motif in the A region of the C-tail did not appear to be required for viability, as indicated by rescue with UAS-dinr-LESL and with UAS-dinr-LESL,Y2F, the latter showing 
similar rescue potential to the Y2 mutation alone. Mutation of the juxtamembrane NPFY tyrosine alone had little effect on viability as DInR-JM-NPFF rescued lethality substantially. Mutations in individual or multiple candidate Chico binding sites, DInRY9F and DInR-Y7,8,9,10F rescued lethality to a lesser degree. Finally, DInR-5NPXF, a compound variant containing mutations of the juxtamembrane NPFY tyrosine and all candidate Chico binding sites in the C-tail rescued viability. In sum, most DInR proteins carrying small deletions and specific mutations retained the ability to complement loss of DInR function, rescuing mutants to adulthood. The exceptions to this were DInR- $\triangle \mathrm{AB}$ and DInR-Y1F. Since the latter targets only one tyrosine residue in the A region of the $\mathrm{C}$-tail and was required for viability, this probably accounts for the failure of DInR- $\triangle \mathrm{AB}$ to rescue lethality.

\section{CHICO INTERACTION SITES ARE REQUIRED FOR DInR'S GROWTH FUNCTION}

We next investigated the size of animals rescued by different DInR variants, with the expectation that animals carrying mutations in Dock binding sites would be similar in size to animals rescued by full-length DInR, while those lacking Chico interaction sites would be similar to small chico mutants. As shown in Figure 4A, the size of male transheterozygotes expressing fulllength DInR was similar to that of sibling heterozygote controls expressing full-length DInR (dinr, $S b$ ). Mutations in the putative Dock binding sites did not have detrimental effects on growth; DInR-LESL and DInR-Y2F rescued adult male growth, although DInR-LESL,Y2F animals were somewhat smaller. Mutation of the juxtamembrane NPFY tyrosine (DInR-JM-NPFF) led to an incomplete rescue of growth (note that an average of two independent lines is shown for this transgene; for all other transgenes, results are shown for a single transformant line). Mutation of one or more Chico binding sites in the C-tail did not have detrimental effects on growth: DInR-Y9F, DInR-Y10F and DInR-Y7,8,9,10F fully rescued growth defects, as did DInR- $\Delta \mathrm{CD}$, which lacks the C-terminal half of the C-tail containing Y7, 8, 9, and 10. However, there was a $50 \%$ decrease in the mean mass of adult males rescued by DInR-5NPXF compared to those rescued by the DInR control. This decrease is very similar to the 55\% decrease in body weight seen in chico mutant males (Bohni et al., 1999). Thus, it seems likely that these five NPXY sites are responsible for most, if not all, of the control of growth by DInR that is mediated by Chico, and that there is considerable redundancy among these NPXY sites.

In females, there was a $52 \%$ decrease in the mean mass of adult females rescued by DInR-5NPXF compared to those rescued by the control DInR protein (Figures $4 \mathrm{~B}, \mathrm{C}$ ). This was noticeably less than the $65 \%$ decrease in body weight measured in chico mutant females (Bohni et al., 1999). Interestingly, although the DInR$\triangle \mathrm{CD}$ protein, which lacks the four NPXY sites in the C-tail in addition to two other tyrosines and one PXXP site, rescued the mean mass of adult males to the same level as the control DInR protein, this did not occur for adult females; instead, there was a $23 \%$ decrease in mean mass in females rescued by the DInR$\triangle \mathrm{CD}$ protein. Thus, it is possible that another interaction site(s) in regions $\mathrm{C}$ and $\mathrm{D}$, other than the four NPXY sites, may also be
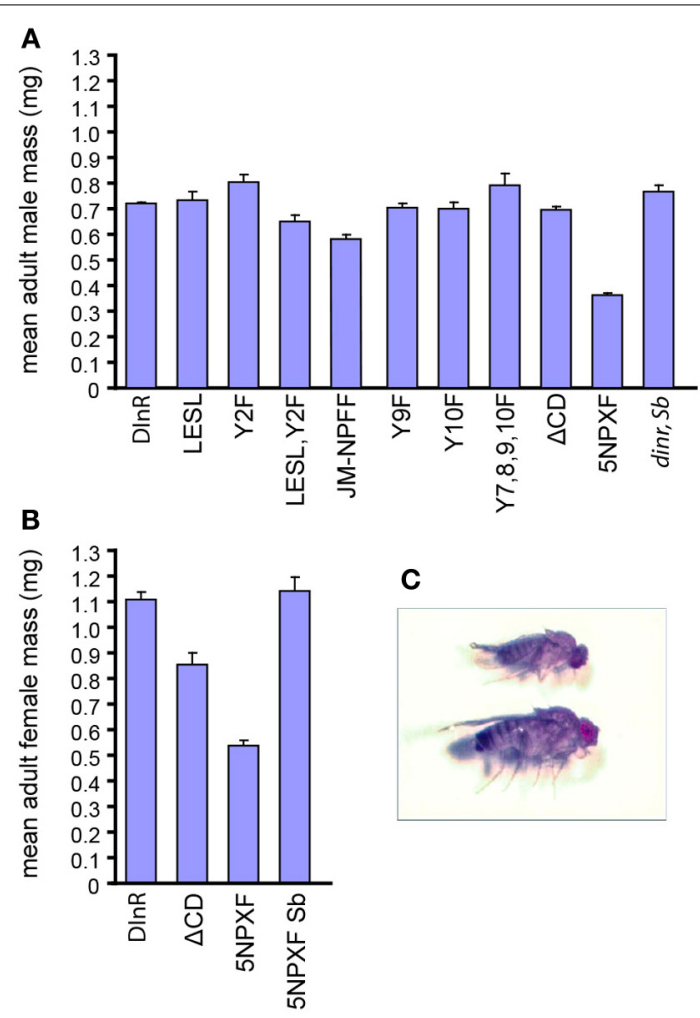

FIGURE 4 | Chico interaction sites are required for whole animal growth. (A,B) Mean mass (mg) of male (A) and female (B) adult dinrex15/273 flies expressing different UAS-DInR variants. Error bars represent standard error. Sample numbers for each rescue protein tested in males were: DInR control, 85; DInR-LESL, 10; DInR-Y2F, 5; DInR-LESL,Y2F, 4; DInR-JM-NPFF, 33; DInR-Y9F, 10; DInR-Y10F, 5; DInR-Y7,8,9,10F, 2; DInR- $\triangle C D$, 27; DInR-5NPXF, 24; DInR control expressed in dinr mutant heterozygote siblings, 4 . Sample numbers for each rescue protein tested in females were: DInR control, 32; DInR- $\triangle$ CD, 8; DInR-5NPXF, 9; DInR-5NPXF expressed in dinr mutant heterozygote siblings, 3. (C) Photograph showing an example of a small dinr mutant adult fly expressing DInR-5NPXF (top) compared to heterozygous sibling control expressing DInR-5NPXF (bottom).

responsible for growth control through Chico in females. Since DInR is also involved in controlling female fertility [reviewed in Garofalo (2002)], an interplay between fertility and growth may be at work.

\section{MUTATION OF CHICO INTERACTION SITES DID NOT ABOLISH RESCUE OF dinr-ASSOCIATED AXON GUIDANCE DEFECTS}

As shown in Figure 5, severe axon guidance defects were observed in dinr ${ }^{e x 15 / 273}$ transheterozygotes, which display gross disorganization of photoreceptor axon targeting in both the lamina and medulla (compare wild type eye-brain complexes, Figures 5A,B, to dinrex15/273 eye-brain complexes, Figures 5C,D). Clumps of axons were present above and in the lamina, and in the medulla.

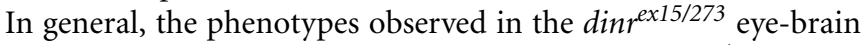
complexes were more severe than those of $\operatorname{dinr}^{353 / 273}$ (Song et al., 2003). This is consistent with dinrex15, a null allele (Song et al., 2003), being a stronger loss-of-function allele than $\operatorname{dinr}^{353}$. Importantly, these axon guidance defects were largely rescued 

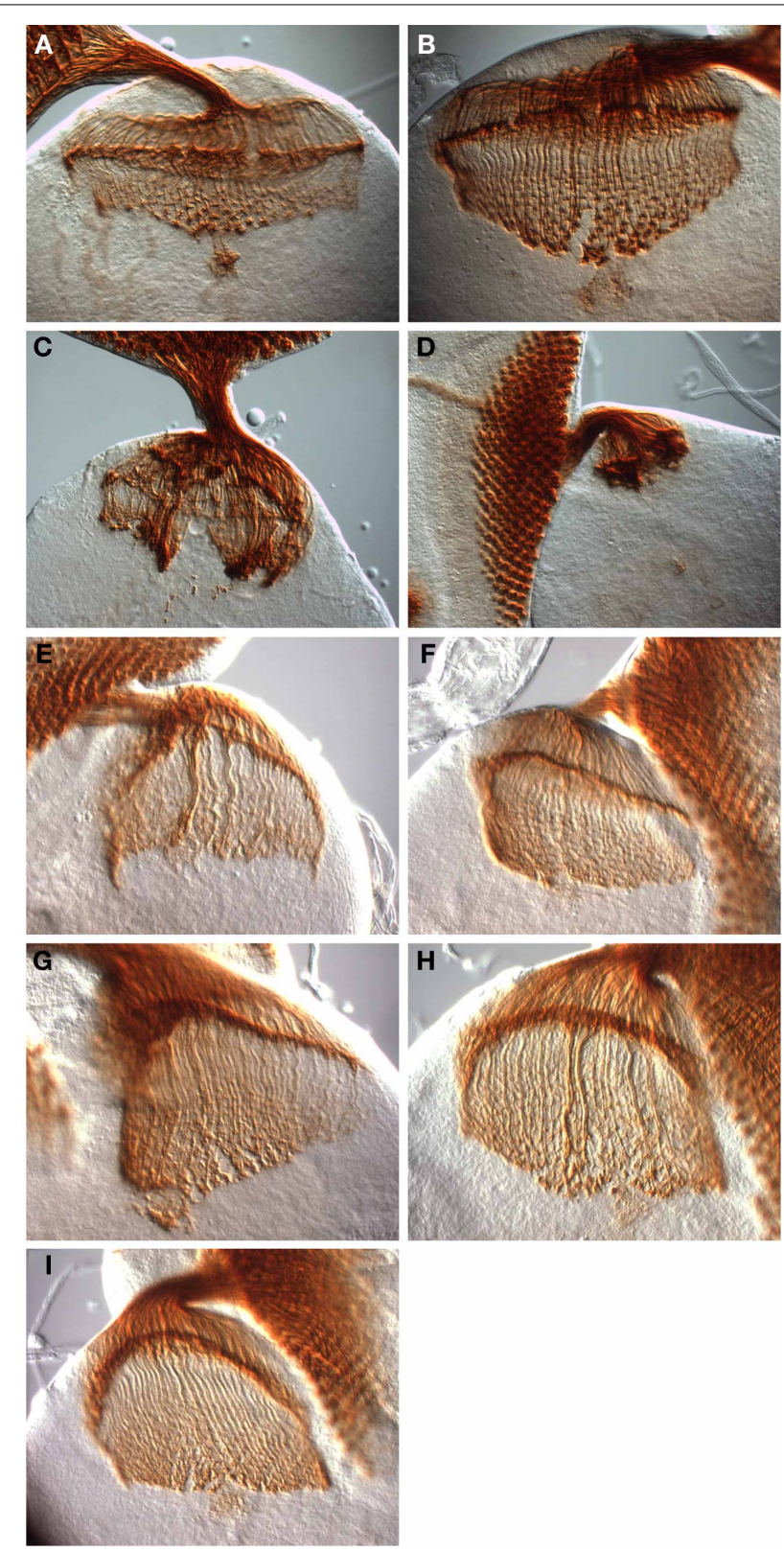

FIGURE 5 | Rescue of dinr-associated axon guidance defects by full-length DInR and variants. Third instar larval eye-brain complexes were stained with MAb24B10 to visualize differentiated photoreceptors and their axons. Imaging was done with DIC optics and a $40 \times$ objective. (A,B) Control eye-brain complexes of the genotype $w^{1118}$. The specimen shown in (B) is at a later stage of development than that shown in (A). The rip in the medulla in (B) resulted during sample preparation. (C,D) Axon guidance was severely disrupted in eye-brain complexes from whole animal dinrex15/273 transheterozygotes. (E) Axon guidance defects were rescued with full-length DInR. (F-I) Axon guidance defects were rescued by expression of DInR variants: (F) DInR-LESL; (G) DInR-Y2F; (H) DInR-LESL, Y2F; (I) DInR-5NPXF.

by expression of full-length DInR (Figure 5E). This is the first demonstration that axon guidance defects are rescued by DInR, ruling out the possibility that defects seen in dinr mutants result from the genetic background of the dinr mutants. Surprisingly, the DInR variants carrying mutations in candidate Dock binding sites also rescued axon guidance defects. These include DInRLESL (Figure 5F), in which one of the putative Dock binding sites was mutated, DInR-Y2F (Figure 5G), in which the second putative Dock binding site was mutated, and DInR-LESL, Y2F (Figure 5H), in which both putative Dock binding sites were mutated. Notably, DInR-5NPXF, which did not rescue growth defects (see above, Figure 4), did restore normal photoreceptor axon guidance (Figure 5I). This rescue by DInR-5NPXF demonstrates that Chico interaction sites are not required for axon guidance functions of DInR. Furthermore, this shows that dinrassociated axon guidance defects are not merely a secondary effect of dinr-associated growth defects.

\section{CONCLUSIONS}

Like other RTKs, DInR regulates multiple processes, including growth and axon guidance. Here we have tested the hypothesis that DInR interacts differentially in vivo with different adapter proteins to mediate different biological functions. In cell-based assays, different regions of DInR interact with Chico and Dock, adapter proteins implicated in growth and axon guidance, respectively. Using in vivo rescue experiments, we found that mutations in DInR's Chico interaction sites rescued viability and axon guidance defects, but, as expected, animals were small, similar to chico mutants. This finding supports the notion that DInR interacts directly with Chico to control growth during Drosophila development. In contrast, expression of both wild type and mutant DInR proteins rescued axon guidance defects of dinr mutants. Rescue by wild type DInR confirmed its role in photoreceptor axon targeting. However, it was not expected that DInR variants with mutations in Dock binding sites (Y2, LESL) would rescue axon guidance. At first glance, these results appear to suggest that Dock interaction with DInR is not required for DInR function in axon guidance. However, several other explanations are possible. For example, the single tyrosine identified in our studies that is required for viability (Y1) may also be required for DInR's role in axon guidance. Alternatively, Dock may be capable of binding to multiple sites in DInR in vivo, possibly including the many candidate tyrosines and PXXPs in the C-tail (Figure 1B). This would lead to functional redundancy in vivo, providing a high degree of buffering to ensure interaction between the two proteins and proper axon targeting. In sum, our studies demonstrate that DInR utilizes different protein domains, and likely different adapter proteins, to segregate signaling in axon guidance and growth.

\section{AUTHOR CONTRIBUTIONS}

Caroline R. Li and Dongyu Guo designed and carried out experiments and analyzed data. Leslie Pick designed experiments and oversaw experiments. Leslie Pick and Caroline R. Li wrote the paper.

\section{ACKNOWLEDGMENTS}

The Bloomington Drosophila Stock Center provided fly stocks. We are grateful to Chi-Hon Lee and Jian Wang for helpful suggestions throughout and for sharing unpublished reagents. We thank Mike Ketteringham and Julie Hou for technical assistance, Hua Zhang for sequencing assistance, Amanda Field for 
comments on the manuscript, and the National Institutes of Health (R01 EY14290) and the March of Dimes Birth Defects Foundation for support. Leslie Pick acknowledges support from a Summer Fellowship from the General Research Board, University of Maryland. Caroline R. Li received partial support from a Wayne T. and Mary T. Hockmeyer Endowed Fellowship and an Ann G. Wylie Dissertation Fellowship, both from the University of Maryland.

\section{REFERENCES}

Baserga, R., Peruzzi, F., and Reiss, K. (2003). The IGF-1 receptor in cancer biology. Int. J. Cancer 107, 873-877. doi: 10.1002/ijc.11487

Bateman, J. M., and McNeill, H. (2004). Temporal control of differentiation by the insulin receptor/tor pathway in Drosophila. Cell 119, 87-96. doi: 10.1016/j.cell.2004.08.028

Belgacem, Y. H., and Martin, J. R. (2006). Disruption of insulin pathways alters trehalose level and abolishes sexual dimorphism in locomotor activity in Drosophila. J. Neurobiol. 66, 19-32. doi: 10.1002/neu.20193

Bohni, R., Riesgo-Escovar, J., Oldham, S., Brogiolo, W., Stocker, H., Andruss, B. F., et al. (1999). Autonomous control of cell and organ size by CHICO, a Drosophila homolog of vertebrate IRS1-4. Cell 97, 865-875. doi: 10.1016/S00928674(00)80799-0

Brand, A. H., Manoukian, A. S., and Perrimon, N. (1994). Ectopic expression in Drosophila. Methods Cell Biol. 44, 635-654. doi: 10.1016/S0091679X(08)60936-X

Britton, J. S., Lockwood, W. K., Li, L., Cohen, S. M., and Edgar, B. A. (2002). Drosophila's insulin/PI3-kinase pathway coordinates cellular metabolism with nutritional conditions. Dev. Cell 2, 239-249. doi: 10.1016/S15345807(02)00117-X

Brogiolo, W., Stocker, H., Ikeya, T., Rintelen, F., Fernandez, R., and Hafen, E. (2001). An evolutionarily conserved function of the Drosophila insulin receptor and insulin-like peptides in growth control. Curr. Biol. 11, 213-221. doi: 10.1016/S0960-9822(01)00068-9

Chen, C., Jack, J., and Garofalo, R. S. (1996). The Drosophila insulin receptor is required for normal growth. Endocrinology 137, 846-856. doi: 10.1210/en.137.3.846

Coelho, C. M., and Leevers, S. J. (2000). Do growth and cell division rates determine cell size in multicellular organisms? J. Cell Sci. 113(Pt 17), 2927-2934.

Colombani, J., Bianchini, L., Layalle, S., Pondeville, E., Dauphin-Villemant, C., Antoniewski, C., et al. (2005). Antagonistic actions of ecdysone and insulins determine final size in Drosophila. Science 310, 667-670. doi: 10.1126/science. 1119432

Copps, K. D., and White, M. F. (2012). Regulation of insulin sensitivity by serine/threonine phosphorylation of insulin receptor substrate proteins IRS1 and IRS2. Diabetologia 55, 2565-2582. doi: 10.1007/s00125-012-2644-8

Dickson, B. J. (2003). Development. wiring the brain with insulin. Science 300, 440-441. doi: 10.1126/science. 1084513

Fernandez, R., Tabarini, D., Azpiazu, N., Frasch, M., and Schlessinger, J. (1995). The Drosophila insulin receptor homolog: a gene essential for embryonic development encodes two receptor isoforms with different signaling potential. EMBO J. 14, 3373-3384.

Fernandez-Almonacid, R., and Rosen, O. M. (1987). Structure and ligand specificity of the Drosophila melanogaster insulin receptor. Mol. Cell. Biol. 7, 2718-2727.

Fisher, T. L., and White, M. F. (2004). Signaling pathways: the benefits of good communication. Curr. Biol. 14, R1005-R1007. doi: 10.1016/j.cub.2004.11.024

Garofalo, R. S. (2002). Genetic analysis of insulin signaling in Drosophila. Trends Endocrinol. Metab. 13, 156-162. doi: 10.1016/S1043-2760(01)00548-3

Garrity, P. A., Rao, Y., Salecker, I., McGlade, J., Pawson, T., and Zipursky, S. L. (1996). Drosophila photoreceptor axon guidance and targeting requires the dreadlocks SH2/SH3 adapter protein. Cell 85, 639-650. doi: 10.1016/S00928674(00)81231-3

Giannakou, M. E., Goss, M., and Partridge, L. (2008). Role of dFOXO in lifespan extension by dietary restriction in Drosophila melanogaster: not required, but its activity modulates the response. Aging Cell 7, 187-198. doi: 10.1111/j.14749726.2007.00362.x
Goberdhan, D. C., and Wilson, C. (2003). The functions of insulin signaling: size isn't everything, even in Drosophila. Differentiation 71, 375-397. doi: 10.1046/j.1432-0436.2003.7107001.x

Hou, J. C., and Pessin, J. E. (2007). Ins (endocytosis) and outs (exocytosis) of GLUT4 trafficking. Curr. Opin. Cell Biol. 19, 466-473. doi: 10.1016/j.ceb.2007.04.018

Hsu, H. J., LaFever, L., and Drummond-Barbosa, D. (2008). Diet controls normal and tumorous germline stem cells via insulin-dependent and independent mechanisms in Drosophila. Dev. Biol. 313, 700-712. doi: 10.1016/j.ydbio.2007.11.006

Hubbard, S. R. (2013). The insulin receptor: both a prototypical and atypical receptor tyrosine kinase. Cold Spring Harb. Perspect. Biol. 5, a008946. doi: 10.1101/cshperspect.a008946

Hwangbo, D. S., Gershman, B., Tu, M. P., Palmer, M., and Tatar, M. (2004). Drosophila dFOXO controls lifespan and regulates insulin signalling in brain and fat body. Nature 429, 562-566. doi: 10.1038/nature02549

Kahn, R. C., King, G. L., Moses, A. C., Weir, G. C., Jacobson, A. M., and Smith, R. J. (2005). Joslin's Diabetes Mellitus, 14th Edn. Boston, MA: Lippincott Williams \& Wilkins.

Kholodenko, B. N. (2006). Cell-signalling dynamics in time and space. Nat. Rev. Mol. Cell Biol. 7, 165-176. doi: 10.1038/nrm1838

Kohanski, R. A. (2007). "Insulin receptor," in Diabetes and Carbohydrate Metabolism, eds I. D. Goldfine and R. J. Rushakoff. Available online at: http://www.endotext.org/diabetes/index.htm

LaFever, L., and Drummond-Barbosa, D. (2005). Direct control of germline stem cell division and cyst growth by neural insulin in Drosophila. Science 309, 1071-1073. doi: 10.1126/science. 1111410

Leevers, S. J. (2001). Growth control: invertebrate insulin surprises! Curr. Biol. 11, R209-R212. doi: 10.1016/S0960-9822(01)00107-5

Lemmon, M. A., and Schlessinger, J. (2010). Cell signaling by receptor tyrosine kinases. Cell 141, 1117-1134. doi: 10.1016/j.cell.2010.06.011

Lingo, P. R., Zhao, Z., and Shen, P. (2007). Co-regulation of coldresistant food acquisition by insulin- and neuropeptide Y-like systems in Drosophila melanogaster. Neuroscience 148, 371-374. doi: 10.1016/j.neuroscience.2007.06.010

Mair, W., Piper, M. D., and Partridge, L. (2005). Calories do not explain extension of life span by dietary restriction in Drosophila. PLoS Biol. 3:e223. doi: 10.1371/journal.pbio.0030223

Marin-Hincapie, M., and Garofalo, R. S. (1999). The carboxyl terminal extension of the Drosophila insulin receptor homologue binds IRS-1 and influences cell survival. J. Biol. Chem. 274, 24987-24994. doi: 10.1074/jbc.274.35.24987

Min, K. J., Yamamoto, R., Buch, S., Pankratz, M., and Tatar, M. (2008). Drosophila lifespan control by dietary restriction independent of insulin-like signaling. Aging Cell 7, 199-206. doi: 10.1111/j.1474-9726.2008.00373.x

Murphy, L. O., and Blenis, J. (2006). MAPK signal specificity: the right place at the right time. Trends Biochem. Sci. 31, 268-275. doi: 10.1016/j.tibs.2006.03.009

Nakae, J., Kido, Y., and Accili, D. (2001). Tissue-specific insulin resistance in type 2 diabetes: lessons from gene-targeted mice. Ann. Med. 33, 22-27. doi: 10.3109/07853890109002056

Newsome, T. P., Asling, B., and Dickson, B. J. (2000). Analysis of Drosophila photoreceptor axon guidance in eye-specific mosaics. Development 127, 851-860.

Ocorr, K., Perrin, L., Lim, H. Y., Qian, L., Wu, X., and Bodmer, R. (2007). Genetic control of heart function and aging in Drosophila. Trends Cardiovasc. Med. 17, 177-182. doi: 10.1016/j.tcm.2007.04.001

Oldham, S., Stocker, H., Laffargue, M., Wittwer, F., Wymann, M., and Hafen, E. (2002). The Drosophila insulin/IGF receptor controls growth and size by modulating PtdInsP(3) levels. Development 129, 4103-4109.

Partridge, L. (2008). Some highlights of research on aging with invertebrates. Aging Cell 7, 605-608. doi: 10.1111/j.1474-9726.2008.00415.x

Pawson, T. (2007). Dynamic control of signaling by modular adaptor proteins. Curr. Opin. Cell Biol. 19, 112-116. doi: 10.1016/j.ceb.2007.02.013

Pessin, J. E., and Frattali, A. L. (1993). Molecular dynamics of insulin/IGF-I receptor transmembrane signaling. Mol. Reprod. Dev. 35, 339-344. discussion: 344-345. doi: 10.1002/mrd.1080350404

Petruzzelli, L., Herrera, R., Arenas-Garcia, R., Fernandez, R., Birnbaum, M. J., and Rosen, O. M. (1986a). Isolation of a Drosophila genomic sequence homologous to the kinase domain of the human insulin receptor and detection of the phosphorylated Drosophila receptor with an anti-peptide antibody. Proc. Natl. Acad. Sci. U.S.A. 83, 4710-4714. doi: 10.1073/pnas.83.13.4710 
Petruzzelli, L., Herrera, R., Garcia-Arenas, R., and Rosen, O. M. (1986b). Acquisition of insulin-dependent protein tyrosine kinase activity during Drosophila embryogenesis. J. Bio. Chem. 260, 16072-16075.

Piper, M., Mair, W., and Partridge, L. (2007). Comment by Matthew Piper, William Mair, Linda Partridge on Min, K. J., Flatt, T., Kulaots, I., Tatar, M. (2006). "Counting calories in Drosophila dietary restriction" Exp. Gerontology. Exp. Gerontol. 42, 253-255. doi: 10.1016/j.exger.2006.10.009

Piper, M. D., Mair, W., and Partridge, L. (2005). Counting the calories: the role of specific nutrients in extension of life span by food restriction. J. Gerontol. A Biol. Sci. Med. Sci. 60, 549-555. doi: 10.1093/gerona/60.5.549

Piper, M. D., and Partridge, L. (2007). Dietary restriction in Drosophila: delayed aging or experimental artefact? PLoS Genet. 3:e57. doi: 10.1371/journal.pgen.0030057

Poltilove, R. M., Jacobs, A. R., Haft, C. R., Xu, P., and Taylor, S. I. (2000). Characterization of Drosophila insulin receptor substrate. J. Biol. Chem. 275, 23346-23354. doi: 10.1074/jbc.M003579200

Poy, M. N., Yang, Y., Rezaei, K., Fernstrom, M. A., Lee, A. D., Kido, Y., et al. (2002). CEACAM1 regulates insulin clearance in liver. Nat. Genet. 30, 270-276. doi: $10.1038 / \mathrm{ng} 840$

Puig, O., and Tjian, R. (2006). Nutrient availability and growth: regulation of insulin signaling by dFOXO/FOXO1. Cell Cycle 5, 503-505. doi: $10.4161 /$ cc.5.5.2501

Rao, Y., and Zipursky, S. L. (1998). Domain requirements for the dock adapter protein in growth-cone signaling. Proc. Natl. Acad. Sci. U.S.A. 95, 2077-2082. doi: 10.1073/pnas.95.5.2077

Ronshaugen, M., McGinnis, N., and McGinnis, W. (2002). Hox protein mutation and macroevolution of the insect body plan. Nature 415, 914-917. doi: 10.1038/nature716

Ruan, Y., Chen, C., Cao, Y., and Garofalo, R. S. (1995). The Drosophila insulin receptor contains a novel carboxy-terminal extension likely to play an important role in signal transduction. J. Biol. Chem. 270, 4236-4243. doi: 10.1074/jbc.270.9.4236

Shingleton, A. W., Das, J., Vinicius, L., and Stern, D. L. (2005). The temporal requirements for insulin signaling during development in Drosophila. PLoS Biol. 3:e289. doi: 10.1371/journal.pbio.0030289

Song, J., Wu, L., Chen, Z., Kohanski, R. A., and Pick, L. (2003). Axons guided by insulin receptor in Drosophila visual system. Science 300, 502-505. doi: $10.1126 /$ science. 1081203

Taguchi, A., and White, M. F. (2008). Insulin-like signaling, nutrient homeostasis, and life span. Annu. Rev. Physiol. 70, 191-212. doi: 10.1146/annurev.physiol.70.113006.100533
Tatar, M. (2004). The neuroendocrine regulation of Drosophila aging. Exp. Gerontol. 39, 1745-1750. doi: 10.1016/j.exger.2004.06.024

Tatar, M., Kopelman, A., Epstein, D., Tu, M. P., Yin, C. M., and Garofalo, R. S. (2001). A mutant Drosophila insulin receptor homolog that extends life-span and impairs neuroendocrine function. Science 292, 107-110. doi: 10.1126/science. 1057987

White, M. F. (1998). The IRS-signalling system: a network of docking proteins that mediate insulin action. Mol. Cell. Biochem. 182, 3-11. doi: 10.1023/A:1006806722619

Wu, Q., Zhao, Z., and Shen, P. (2005). Regulation of aversion to noxious food by Drosophila neuropeptide Y- and insulin-like systems. Nat. Neurosci. 8, 1350-1355. doi: 10.1038/nn1540

Yenush, L., Fernandez, R., Myers, M. G. J., Grammer, T. C., Sun, X. J., Blenis, J., et al. (1996). The Drosophila insulin receptor activates multiple signaling pathways but requires insulin receptor substrate proteins for DNA synthesis. Mol. Cell. Biol. 16, 2509-2517.

Zipursky, S. L., Venkatesh, T. R., Teplow, D. B., and Benzer, S. (1984). Neuronal development in the Drosophila retina: monoclonal antibodies as molecular probes. Cell 36, 15-26. doi: 10.1016/0092-8674(84)90069-2

Conflict of Interest Statement: The authors declare that the research was conducted in the absence of any commercial or financial relationships that could be construed as a potential conflict of interest.

Received: 30 September 2013; accepted: 09 December 2013; published online: 20 January 2014.

Citation: Li CR, Guo D and Pick L (2014) Independent signaling by Drosophila insulin receptor for axon guidance and growth. Front. Physiol. 4:385. doi: 10.3389/fphys. 2013.00385

This article was submitted to Invertebrate Physiology, a section of the journal Frontiers in Physiology.

Copyright (c) $2014 \mathrm{Li}$, Guo and Pick. This is an open-access article distributed under the terms of the Creative Commons Attribution License (CC BY). The use, distribution or reproduction in other forums is permitted, provided the original author(s) or licensor are credited and that the original publication in this journal is cited, in accordance with accepted academic practice. No use, distribution or reproduction is permitted which does not comply with these terms 